externum by the continuance of the same pain. Having hastily tied and divided the funis, and removed the placenta, (which was perfectly loose,) I passed my finger into the vagina to ascertain the condition of the os uteri, which I could feel high up, widely dilated, and embracing a soft, globular substance, which protruded through it, and occupied the vagina. This I at first imagined was the bag of membranes belonging to the other foetus I had suspected to be there. To satisfy myself, I placed my other hand on the abdomen, but, to my surprise, could feel nothing like the uterus there, although I made deep pressure for it. I therefore instituted a more careful examination of the tumour itself, when the following circumstances assisted me in forming a diagnosis. First. It was exceedingly sensitive; the least movement of my finger across it occasioned her great pain. Second. Hæmorrhage commenced, though not to any alarming extent. Third. Symptoms of prostration suddenly manifested themselves, more than I should have expected, either from the sudden delivery, or from the bleeding. I accordingly concluded that the present must be a case of partial inversion of the uterus, the fundus having followed, by its sudden and energetic contraction, the placenta, through the os ateri, whilst this latter remained dilated.

Acting under this conclusion, I pressed the knucleles of two fingers against the most dependent part of the tumour, and was delighted to feel it gradually giving way, until it suddenly started back into its place in the pelvis, leaving my hand occupying its cavity. I could now distinctly feel it through the abdominal parietes, firm and well contracted, although the os uteri still remained considerably dilated. The hæmorrhage immediately ceased when the inversion was reduced. After waiting an hour with her, during which time there was no symptom indicating danger, and having applied a bandage firmly round the lower part of the abdomen, I left her. Mrs. C__ had a speedy and most favourable recovery.

There are a few circumstances connected with the foregoing history that are perhaps worthy of being considered more in detail. First. This was evidently a case of spontaneous inversion, produced by the vigorous but irregular contraction of the uterus. It is undoubtedly true, that by far the greater proportion of these cases, when they do occur, arise from unskilful management in the last stage of labour, unjustifiable traction being made at the funis, for the purpose of speedily removing the placenta; whilst the uterus itself remains dilated and flaccid, so that the fundus is literally dragged down through the os internum into the vagina, where it is but too often allowed to remain, until all possibility of returning it is for ever lost. Still I think it may be fairly questioned whether traction is always the exciting cause of this accident, whether made by the hand of the accoucheur, or by the unusual shortness of the cord pulling down the placenta and fundus uteri, where it has occurred in cases of very rapid delivery : at any rate, neither of these causes could operate in the case I have related; for, in the first place, I had no opportunity of removing the placenta from the uterus, and, in the second, the funis was longer thau ordinary-a point concerning which I fully satisfied myself before leaving the room.

Every experienced accoucheur is aware that irregularities do frequently occur in the contraction of the uterus, one part becoming hard and firm, whilst another remains soft and dilated; in fact, it is from this very circumstance that so much difficulty is occasionally experienced in removing a retained placenta. I have frequently observed, especially in thin women, in whom the uterus could be distinctly felt through the abdominal walls, that, during its contractions, after the expulsion of the child, there has been for a short period a very manifest depression in one situition, but which, immediately afterwards, I have been anable to find. This circumstance, I imagine, depends upon some irregularity or inequality in the action of the uterine fibres; and the same cause acting more powerfully, particularly at the fundus of the uterus, may undoubtedly give rise to inversion. Nor is this mere speculation, for accidents of this kind now and then happen, which cannot be satisfactorily explained upon any other hypothesis; as, for example, in those cases where the child has been expelled after the death of the mother, and where the process has not been in the slightest degree interfered with.* Again, cases of inversion are recorded where, for several hours and even days after delivery, no symptom has manifested itself which could lead even the most anxious attendant to suspect the occurrence of such a fearful accident. A case is quoted in the 51st vol. of The London Medical and Physical Journal, of a woman who was rapidly recovering from a favourable labour, but who, on the tenth day from her delivery, "whilst making

- See cases referred to in the Edinburgh Medical and Surgical Journal for January, 1840 . violent efforts to evacuate the contents of the rectum, felt a bulk mass descend through the vagina, which was followed by great pain in the belly and the displaced parts, and in the groin, with strong efforts to vomit, and a sensation of faintness." The uterus was discovered to be completely inverted, and the accident had undoubtedly occurred when the straining efforts were made One of the most intelligent practitioners in Birmingham, who has deservedly enjoyed a large midwifery practice, has informed me that he once attended a woman in her labour, who was favourably recovering, having had no bad symptom, when, on the second day from her confinement, the uterus was inverted during the operation of a quantity of castor oil, which she had taken without his lsnowledge.

Other cases might be adduced of the same character, and the conclusion that forces itself upon the nind is, that inversion does sometimes take place unconnected with any traction made on the fundus uteri, either by an unusually short funis, or by the hand of the accoucheur.

Second. Of the symptoms present in the above case, two were most prominent-viz., the highly sensitive tumour occupying the upper part of the vagina, and the sudden depression of the patient. When these symptoms appear after a labour, there can be little doubt as to the nature of the accident, and the necessity for immediate and judicious treatment. I find, upon reference to the published cases of this kind, that in almost every instance, mention is made of the sudden prostration of the vital powers, as indicated by a tendency to syncope, hiccup, \&c. This, I believe, does not arise so much from the loss of blood, as from the shock produced by the sudden displacement of the parts; it is, in fact, similar to the prostration consequent upon injury inflicted upon any other organ largely supplied with the nerves of organic life.

It is not necessary to make any remark upon the first-mentioned symptom, since the discovery of a highly sensitive tumour in such a situation, and at such a time, cannot be mistaken : but I may perhaps express an opinion, that it is incumbent on every one who makes himself responsible for a woman's safety during her labour, not to leave the room until he bas carefully ascertained the condition of the os uteri, and also the situation and degree of contraction of the uterus itself, the former by an examination per vaginam, and the latter through the abdominal ralls.

Third. In no case is the importance of forming a correct diarnosis more forcibly exhibited than in one of this nature. Discovered at the time of its occurrence, nothing is more simple than its reduction, nothing more certain than its successful issue. But let the nature of the case be overlooked or misunderstood in the first instance, and it is almost equally certain that the discovery will be made too late to sare the woman from an untimely and miserable end.

Paradise-street, Birmingham, Sept. 30, 1814

\section{ON THE TREATMENT OF INDOLENT AND} IRRITABLE ULCERS.

By J. Proprck, M.D., late Physician to the St. Giles's Dispensary, London.

INTRACTABLE cases of ulcers on the legs are of frequent occurrence in our hospitals and at our dispensaries. The object of this paper is, to state the mode of treatment which I have found eminently successful in such cases, many of which came under my care during the sixteen years $I$ had the sole charge of the dispensary in St. Giles's. Many of the patients were occupied in selling fish or fruit in the streets; they were standing frequently in the wet, exposed to the weather, from early in the morning till late at night, and the women were employed in washing; their diet was chiefly fish, and that not particularly fresh; ontside rashers of bacon, decayed cheese, and rancid butter; their beverage was tea, porier, and gin; a combination of articles of food highly conducive to impurities of the blood, especially when favoured by a total neglect of personal cleanliness, and by the foul air of their dwellings at night. Under these circumstances, I regarded the nicer on the leg as a natural issue or outlet for the escape of certain morbid principles from the blood, the retention or suppression of which would have occasioned diseases of a more dangerous tendency. Hence, $I$ considered that the popular prejudice against drying up of old ulcers was not devoid of reason.

The analogy between those natural issues in the leg and artificial issues in the arm in affording relief to the system, in cases of disease from impurity of the blood, is very striking.

If the ulcer or the issue emit a disgusting odour, and discharge freely, the necessity for such a drain is unequirocal; it cannot be 
closed without the risk of a worse disorder. But when the odour of the ulcer or the issue ceases to be disagreeable, and the discharge is moderate in quantity, and of a healthy quality, it admits of cure with perfect safety.

The question then arose, of what secretion was the foul discharge from the ulcer vicarious? Not of the bowels, for such patients are not always of a constipated habit, but, on the contrary, they are liable to attacks of diarrhœa; not of the kidney, for the urinary secretion is commonly very abundant; but of the skin, for their diet was of the kind least perspirable, and their occupation most unfavourable for free cutaneous secretion.

Accordingly, after mild purging, (if necessary,) the vapour bath, in which the head of the patient was included, alterative medicines, suitable dressings, and a pure diet, as far as dietetic rules could be enforced among such a class of patients in dispensary practice, were the remedies employed for the safe cure of nlcerated legs.

The aperient medicines were, a small dose (one grain) of calomel and rhubarb at bedtime, and a draught of the white mixture of Epsom salts in mint-water in the morning.

The vapour bath was used for twenty or thirty minutes twice a week.

The alterative medicines were, the decoction of polygala Senega, when a rheumatic diathesis was present, or powdered sarsaparilla in milk, when there was a suspicion of a syphilitic contamination.

The dressings were, warm water, solutions of nitrate of silver, sulphate of copper, or of the chlorides of soda and lime; or dry lint, powdered rhubarb, Peruviau bark, charcoal, or chalk, with light bandaging.

But the vapour bath was the principal remedy, for without the sweating process all the other means were powerless. The perspiration produced by the vaponr bath, the first few times of asing it, exhaled an odour exactly like that of the discharge from the ulcer. After awhile, this odour totally ceased, both from the alcer and from the perspiration; and then, but not before, the wleer began to heal, generally with no other dressing than warmwater and oil-silk, or simply a piece of dry lint, to defend it from external injury.

The elimination and escare of the morbid elements from the blood having been accomplished by the free ettaneous secretion, the necessity no longer existed for their discharge by the ulcer, which therefore bealed almost of its own accord, and with perfect safety to the patient, whose general health was invariably improved by the treatment.

Exposure to the same external causes, and retarning to the same diet, were sometimes followed by the same effects, and after the lapse of a year or two, the same patients applied at the dispensary to be cured of an ulcer on the leg by the same process.

Ulcers on the legs are frequently associated with varicose voins, so that varices and ulcers have been considered as standing in the relation of cause and effect. Hence the attempt to cure the latter by an operation to obliterate the former-an attempt which happily has generally proved abortive; for if it had succeeded, the state of the blood remaining unaltered, the health of the patient probably would have suffered in consequence of stopping a salutary discharge; nor has the practice been altogether unattended with danger from inflammation of the veins and the introduction of pus into the circulation. And, moreover, cases of varix without ulcer are nearly as common among the higher class of society, as cases of varix with ulcer are among the lower, owing to the one class being more exposed to the exciting causes than the other.

Montague-street, Russell-square, Nov. 20, 1844.

\section{BRITISH MEDICAL JOURNALS.}

SINGULAR CASE OF FATAL INTRA-UTERINE HAMORRHAGE.

Mr. Tuompson records, in the Medical Gazette, the case of a female who, having previously borne twelve children, had arrived at the close of the ninth month of pregnancy. As well as usual during the day, she passed a disturbed night. A midwife, sent for on the following morning, found labour commenced, and the presentation natural. The patient gradually became exhausted, and died, undelivered, in a few hours. Examined after death, the uterus was found to be

"Very large, and apparently filling the whole cavity of the abdomen, pressing the floating viscera strongly upwards and backwards; these latter had a very bleached appearance, and all their vessels were empty. On raising the fundus of the uterus, an immense quantity of bloody fluid rushed from the vagina; and on cutting into the womb, (the walls of which were very thin, ) we found that it still contained more than two quarts of fluid and grumous blood, mixed with large coagula, which completely surrounded the child, enveloped in its membranes entire, with the head in the brim of the pelvis.

"The placenta was wholly detached from the uterus, but the place where it had been attached was evident enough, on the right side of the womb, below the fundus: we examined this part very narrowly, but could discover nothing unusual in its appearance, or in that of the placenta."

THE TREATMENT OF TYPHUS FEVER AND ITS COMPLICATIONS.

This subject is discussed at considerable length in the last number of the Edinburgh Monthly Journal, by Dr. Davioson. Plain and practical, without pretensions to novelty, the commum nication is deserving of attention. The author thus expresses his opinions:-

"1. That typhus is a disense which candot be checked in limine, is often tedious in its progress, causing great emaciation and exhaustion; we ought not, therefore, without very strong and special reasons, to employ any measures which may vitally lessen the powers of life, such as bleeding, vomiting, and excessive sweating or purging.

"2. The ordinary measures may be the following:-Place the patient in a large, well-ventilated apartment, on a mattress with few bedclothes; let the head be shaved, and kept cool with an evaporating lotion; give a gentle purgative every second or third day; let the skin be bathed once or twice a day with tepid water, and this may be accompanied with small doses of tartrate of antimony, antimonial powder, or ipecacuan. His drink should be light, cooling, and slightly diuretic, and his diet nutritive, but light, and little liable to ascescency. When there is a tendency to congestion in any organ, a little calomel or hydrargyras c. creta may be combined with the purgative; or calomel, with a small portion of opium, may be given every six or eight hours. The application of two or three leeches to the temples or nostrils is often useful in concestion of the brain, and also when there is intense headache, which is often the forerunner of delirium. Blisters are also often advaistageous in such cases. Derangements of particular functions, or symptoms arising from idiosyncrasy of constitution, sometimes occur, and must be treated accordingly.

" 3. Mercury, in small doses, is frequently useful in promoting several of the secretions, and in relieving the congestions of internal organs.

"4. Opium is injurious in a large proportion of cases, from its tendency to cause congestion in the head; but when diarrhoea is a symptom, it ought to be administered with a view to check the exhausting evacuations.

" 5 . Wines and other alcoholic liquors, as they contain both stimulant and alimentary elements, are the most to be relied on for supporting the strength, and are the least injurious. The pulse, taken along with the general symptoms of exhaustion, ought to be the rule for its [their] exhibition, both as to time and quantity.

"6. Ammonia, camphor, quinine, and other similar tonics, are not to be depended on in bad cases, and when exhibited along with wine, frequently cause the patient to refuse both.

" 7 . When the disease is complicated with local affections in the head, chest, or abdomen, these must be treated on the same general principles as the idiopathic disease, which they represent; with this important modification, that evacuations of all kinds must be employed more sparingly, and with much caution, and that even in these cases, if there be much prostration of strength and a very weak pulse, wine must be administered, although more moderately than in the simple disease."

TANNIN A CURE FOR TOOTHACHE.

Mr. Druitt, in recommending the use of tannin as a remedy in cases of sore nipple, of salivation by mercury, of aphthæ, \&c., says-

"But of all the cases for which it is adapted, that common tronblesome complaint, toothache, is that in which I believe it is most to be depended on. When the tooth aches, let the patient wash out the mouth thoroughly with the solution of carbonate of soda in warm water; let the gum around the tooth, or between it and its neighbours, be scarified with a fine lancet; then let a little bit of cotton wool, imbued with a solution of a scruple of tannin, and five grains of mastich, in two drachms of æther, be put into the eavity, and if the ache is to be cured at all, this plan will put an end to it in nine cases out of ten." 